\title{
Beam diffusion measurements using collimator scans in the LHC
}

\author{
Gianluca Valentino, ${ }^{1,2, *}$ Ralph Aßmann, ${ }^{3, \dagger}$ Roderik Bruce, ${ }^{1}$ Florian Burkart, ${ }^{1}$ Valentina Previtali, ${ }^{4}$ Stefano Redaelli, ${ }^{1}$ \\ Belen Salvachua, ${ }^{1}$ Giulio Stancari, ${ }^{4,}$ and Alexander Valishev ${ }^{4}$ \\ ${ }^{1}$ CERN, Geneva, Switzerland \\ ${ }^{2}$ University of Malta, Msida, Malta \\ ${ }^{3}$ DESY, Hamburg, Germany \\ ${ }^{4}$ Fermi National Accelerator Laboratory, P.O. Box 500, Batavia, Illinois 60510, USA
}

(Received 6 December 2012; published 19 February 2013)

\begin{abstract}
The time evolution of beam losses during a collimator scan provides information on halo diffusion and population. This is an essential input for machine performance characterization and for the design of collimation systems. Beam halo measurements in the CERN Large Hadron Collider were conducted through collimator scrapings in a dedicated beam study for the first time at $4 \mathrm{TeV}$. Four scans were performed with two collimators, in the vertical plane for beam 1 and horizontally for beam 2 , before and after bringing the beams into collisions. Inward and outward steps were performed. A diffusion model was used to interpret the observed loss rate evolution in response to the collimator steps. With this technique, diffusion coefficients were estimated as a function of betatron oscillation amplitude from approximately 3 to 7 standard deviations of the transverse beam distribution. A comparison of halo diffusion and core emittance growth rates is also presented.
\end{abstract}

DOI: 10.1103/PhysRevSTAB.16.021003

PACS numbers: 29.20.dk, 29.27.-a, 05.60.-k

\section{INTRODUCTION}

Understanding particle losses and beam quality degradation is one of the fundamental aspects in the design and operation of accelerators. The dynamics of particles in an accelerator can be quite complex. Deviation from linear dynamics can be large, especially in the beam halo. Lattice resonances and nonlinearities, coupling, intrabeam and beam-gas scattering, as well as the beam-beam force in colliders all contribute to the topology of the particles' phase space, which in general will include regular and chaotic regions, and resonant islands. In addition, various noise sources are present in a real machine, such as ground motion (resulting in orbit and tune jitter) and ripple in the radio-frequency and magnet power supplies. As a result, the macroscopic motion can acquire a stochastic character, which can be described in terms of particle diffusion [1-5].

The LHC consists of eight arcs and eight straight sections, called insertion regions (IRs). The experiments are installed in the insertion points (IPs) of four IRs, where the beams collide. The 100 LHC collimators are arranged in a four-stage hierarchy to scatter and absorb beam halo particles before they are deposited in the superconducting magnets, which may cause quenches [6]. Collimators

\footnotetext{
*gianluca.valentino@cern.ch

${ }^{\dagger}$ On leave from CERN, Geneva, Switzerland.

*stancari@fnal.gov
}

Published by the American Physical Society under the terms of the Creative Commons Attribution 3.0 License. Further distribution of this work must maintain attribution to the author(s) and the published article's title, journal citation, and DOI. also protect sensitive elements and electronics from radiation. The collimators are located mainly in IR3 and IR7 for momentum and betatron cleaning, respectively. There are approximately 3600 beam loss monitor (BLM) ionization chambers installed around the LHC, which measure beam loss rates in Gy/s [7]. A BLM is positioned downstream of each collimator to detect the local losses induced by beam impacts. Throughout this paper, we will often refer to loss rates as "losses" for brevity.

A photograph of a collimator is shown in Fig. 1. Each collimator consists of two blocks or "jaws" of carbon, tungsten, or copper material, which are set symmetrically on either side of the beam to clean halo particles at maximum efficiency. Apart from serving to protect the machine, they may also be used as diagnostic tools, for example to measure the local beam center and beam size in beambased alignment [8], or to serve as sensitive probes of beam orbit jitter [9]. A schematic of the apparatus setup, showing a collimator jaw and a BLM, is provided in Fig. 2.

The time evolution of losses during a collimator scan gives information on halo diffusion, halo population, emittance growth, beam lifetime, and collimation efficiency as a function of collimator position. This study is also useful to check calculations of the dynamic aperture, augment the understanding of present and future collimation systems, and address open questions including the speed and number of particles reaching the collimators. A model capable of predicting the beam losses for a given collimator jaw movement would be an input to establish new algorithms aimed at improving the collimator alignment procedure [10].

A complete set of slow beam scrapings was performed in the $\mathrm{LHC}$ in 2011 at $450 \mathrm{GeV}$ [11]. The measurement 


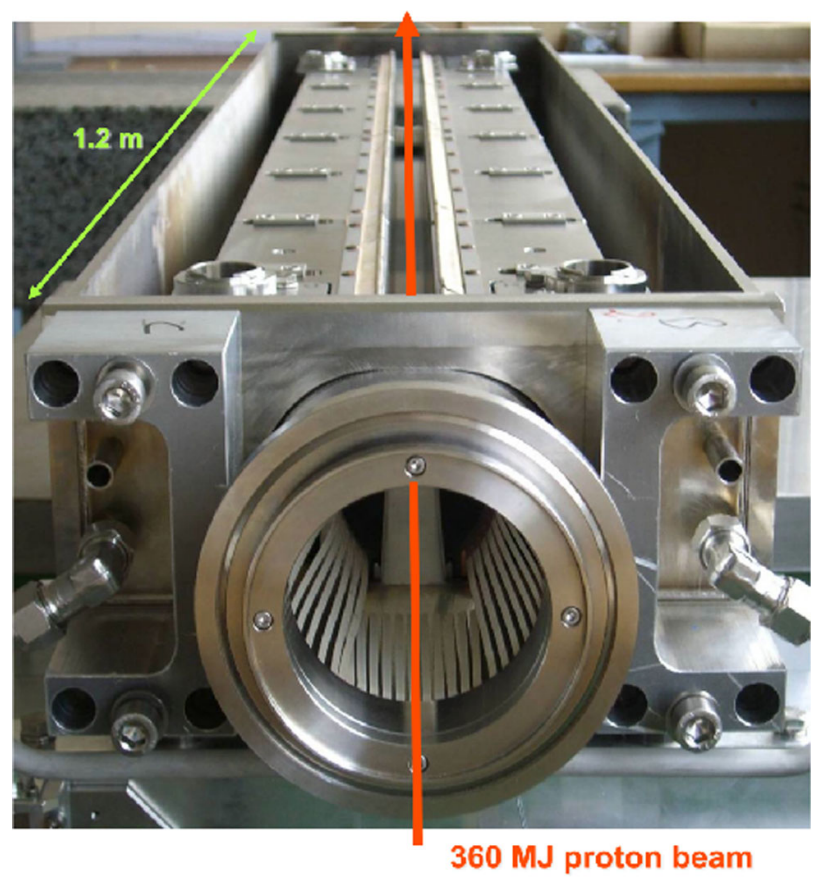

FIG. 1. Photograph of a LHC collimator as seen from one end. The two blocks (jaws) of carbon, tungsten, or copper material need to be placed symmetrically on either side of and parallel to the beam to clean halo particles at maximum efficiency.

data was fitted with a double Gaussian function, and the conclusions of this study were that the horizontal tails are more populated than the vertical tails. A beam study was requested for scraping at $4 \mathrm{TeV}$ following the development of a diffusion model based on data from the Tevatron [12]. The aim of the study is to develop a model based on diffusion to predict the beam losses before, during, and after a collimator step, both inward and outward. These measurements profited from a new $12.5 \mathrm{~Hz}$ BLM data acquisition, whereas only $1 \mathrm{~Hz}$ data was available until 2011. The measurements were done with beams squeezed to the smallest beam sizes used for physics, before and after bringing the beams into collisions, to assess beambeam effects on diffusion.

This paper first explains the diffusion model, which is used to apply the fits to the measured beam loss data. In the following section, the experimental procedure involving the collimator scans is described. The data obtained during

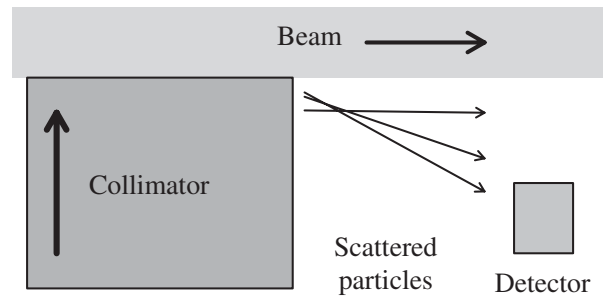

FIG. 2. Schematic of the apparatus setup. The BLM placed downstream of the collimator jaw detects local losses induced by the beam impacts following the movement of the jaw. the study are analyzed to determine intensity-beam loss calibrations, the evolution of beam emittance, and the parametric diffusion model fits. Finally, the results showing comparisons between fits and the measured data are presented.

\section{DIFFUSION MODEL}

Particle motion at the microscopic level is in general very complex. Two main considerations lead to the ansatz that macroscopic motion in a real machine, especially in the halo, will be mostly stochastic: (1) the central limit theorem applied to the multitude of dynamical effects acting on the beam; (2) the operational experience during collimator setup, when spikes and dips in loss rates are generated. These spikes and dips decay in time as $1 / \sqrt{t}$, which is typical of a stochastic diffusion process.

A diffusion model of the time evolution of loss rates caused by a collimator jaw step was developed in [13]. It builds upon the model of Ref. [8] and its assumptions: (1) constant diffusion rate within the range of the step and (2) linear halo distribution tails. These hypotheses allow one to obtain analytical expressions for the solutions of the diffusion equation and for the corresponding loss rates as a function of time. The model in [13] addresses some of the limitations of the previous model and expands it in the following ways: (1) losses before, during, and after the collimator step are predicted; (2) different steady-state rates before and after are accounted for; (3) determination of the model parameters (diffusion coefficient, tail population, detector calibration, and background rate) is more precise and robust against statistical fluctuations in the data, parameter correlations, and initial parameter values in the fits.

These calculations are the basis for the measurement of transverse beam diffusion rates as a function of particle amplitude with collimator scans. Following Ref. [8], we consider the evolution in time $t$ of a beam of particles with phase-space density $f(J, t)$ described by the diffusion equation:

$$
\partial_{t} f=\partial_{J}\left(D \partial_{J} f\right),
$$

where $J$ is the Hamiltonian action and $D$ is the diffusion coefficient. The particle flux at a given location $J=J^{\prime}$ is $\phi=-D \times\left[\partial_{J} f\right]_{J=J^{\prime}}$. During a collimator step, the action limit $J_{c}=x_{c}^{2} / 2 \beta_{c}$, corresponding to the collimator half gap $x_{c}$ at a ring location where the amplitude function is $\beta_{c}$, changes from its initial value $J_{c i}$ to its final value $J_{c f}$ in a time $\Delta t$. The step in action is therefore $\Delta J \equiv J_{c f}-J_{c i}$. In the LHC, typical steps in collimator half gaps $\left(\Delta x_{c}\right)$ are $10 \mu \mathrm{m}$ in $5 \mathrm{~ms}$, and the amplitude function is tens of meters. It is assumed that the collimator steps are small enough so that the diffusion coefficient can be treated as a constant in that region. If $D$ is constant, the local diffusion equation becomes $\partial_{t} f=D \partial_{J J} f$. With these definitions, the particle loss rate at the collimator is equal to the flux at that location: 


$$
L=-D \times\left[\partial_{J} f\right]_{J=J_{c}}
$$

The loss rate evolution measured by the BLMs can be expressed in terms of the particle loss rate $L$, a calibration constant $k$, and a background term $B$ :

$$
S=k L+B
$$

The local losses are proportional to the gradient of the distribution function at the collimator. The value of the gradient at the collimator for inward and outward steps, denoted by the $I$ and $O$ subscripts, respectively, is given by

$$
\begin{aligned}
\partial_{J} f_{I}\left(J_{c}, t\right)= & -A_{i}+2\left(A_{i}-A_{c}\right) P\left(\frac{-J_{c}}{w}\right)-\frac{2 A_{i}\left(J_{c i}-J_{c}\right)}{\sqrt{2 \pi} w} \\
& +\frac{2\left(A_{i} J_{c i}-A_{c} J_{c}\right) e^{\left[-\left(J_{c} / w\right)^{2} / 2\right]}}{\sqrt{2 \pi} w}, \\
\partial_{J} f_{O}\left(J_{c}, t\right)= & -2 A_{i} P\left(\frac{J_{c i}-J_{c}}{w}\right)+2\left(A_{i}-A_{c}\right) P\left(\frac{-J_{c}}{w}\right) \\
& +2 \frac{A_{i} J_{c i}-A_{c} J_{c}}{\sqrt{2 \pi} w} e^{\left[-\left(J_{c} / w\right)^{2} / 2\right]} .
\end{aligned}
$$

The parameters $A_{i}$ and $A_{f}$ are the slopes of the distribution function before and after the step, with $A_{c}$ varying linearly between $A_{i}$ and $A_{f}$ as the collimator moves. The function $P(x)$ is the cumulative Gaussian distribution, and the $w$ term is defined as $w \equiv \sqrt{2 D t}$.

\section{EXPERIMENTAL PROCEDURE}

\section{A. Beam parameters and machine configuration}

The beam parameters, including the beam energy, starting intensity, and $\beta$ functions in the experimental insertion points $\left(\beta^{*}\right)$ for each scraping configuration are shown in Table I. One nominal bunch $\left(1.2 \times 10^{11}\right.$ protons $)$ per beam was used.

\section{B. Beam test program}

The study started off with squeezed, noncolliding beams at an energy of $4 \mathrm{TeV}$. The IR7 primary and secondary collimators were retracted from their nominal settings of $4.3 \sigma$ and $6.3 \sigma$, respectively, to $7 \sigma$ away from the beam center, in order to have a larger scan range. The beam centers at these collimators were measured during beam-

TABLE I. The beam parameters at the start of both scraping configurations.

\begin{tabular}{lcc}
\hline \hline Parameter & Separated beams & Colliding beams \\
\hline Energy $[\mathrm{GeV}]$ & 4000 & 4000 \\
Intensity B1 $\left[10^{11} \mathrm{p}\right]$ & 1.29 & 1.14 \\
Intensity B2 $\left[10^{11} \mathrm{p}\right]$ & 1.07 & 0.74 \\
$\beta^{*} \mathrm{IP} 1 / 5[\mathrm{~cm}]$ & 60 & 60 \\
$\beta^{*} \mathrm{IP} 2 / 8[\mathrm{~cm}]$ & 300 & 300 \\
\hline \hline
\end{tabular}

TABLE II. The settings of the collimators not used for scraping throughout the beam study (grouped by families), for both scraping configurations.

\begin{tabular}{lcc}
\hline \hline Collimator family & Description & Half gap $[\sigma]$ \\
\hline TCP IR3 & Primary collimator & 12 \\
TCSG IR3 & Secondary collimator & 15.6 \\
TCLA IR3 & Absorber & 17.6 \\
TCP IR7 & Primary collimator & 7 \\
TCSG IR7 & Secondary collimator & 7 \\
TCLA IR7 & Absorber & 8.3 \\
TCSG IR6 & Secondary collimator & 7.1 \\
TCDQ IR6 & Dump protection & 7.6 \\
TCT IR1/5 & Tertiary collimator & 9 \\
TCT IR2/8 & Tertiary collimator & 12 \\
TCL IR1/5 & Luminosity debris absorber & Out \\
\hline \hline
\end{tabular}

based alignments in March 2012, and are used throughout the 2012 LHC run. The centers are checked regularly via beam loss maps, and are stable thanks to the reproducibility of the machine. The maximum deviations of the beam centers at the horizontal and vertical primary collimators (TCPs) with respect to the first alignments in the 2012 LHC run were measured to be $160 \mu \mathrm{m}(0.45 \sigma)$ and $110 \mu \mathrm{m}$ $(0.43 \sigma)$, respectively. The beam size is calculated from the nominal beta functions at the individual collimators, with a beam emittance of $3.5 \mu \mathrm{m}$ in both horizontal and vertical planes. An overview of the settings of the collimators not used for scraping during the beam study are shown in Table II. The settings for these collimators, except the TCPs and secondary collimators (TCSGs) in IR7, are the same which are used in normal operation.

In the study, the left jaws of the TCP.D6L7.B1 (vertical plane) and the TCP.C6R7.B2 (horizontal plane) collimators were moved in steps of $5 \mu \mathrm{m}$ to $20 \mu \mathrm{m}$, thus performing a single-sided scraping. The $\beta$ functions and nominal beam sizes at these collimators are provided in Table III. Primary collimators on either side of IR7 were chosen for scraping the counter-rotating beams. Scraping could be done simultaneously for the two beams (see Fig. 3) because the distance between the collimators used for scraping ensured negligible crosstalk between beam loss measurements. For each beam, the jaws were moved in as soon as the beam losses from the previous step had decayed back to a steady state (approximately every 10 to $40 \mathrm{sec}-$ onds). The decay time is inversely proportional to the distance from the beam orbit.

TABLE III. The $\beta$ functions and $1 \sigma$ nominal beam sizes at the collimators used in the scraping.

\begin{tabular}{lrc}
\hline \hline Collimator & $\beta(\mathrm{m})$ & $1 \sigma(\mathrm{mm})$ \\
\hline TCP.D6L7.B1 & 78.26 & 0.253 \\
TCP.C6R7.B2 & 150.36 & 0.351 \\
\hline \hline
\end{tabular}




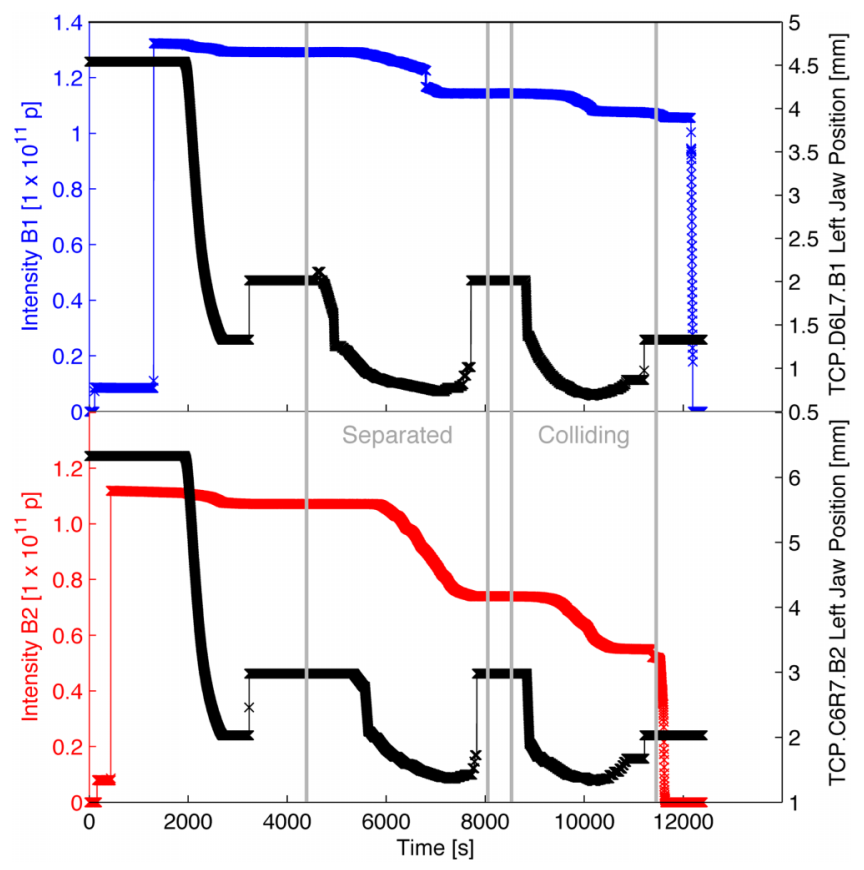

FIG. 3. The beam intensities and the left jaw collimator positions as a function of time (t[0]=22.06.2012, 04:30:00). Scraping with the left jaws of the TCP.D6L7.B1 (vertical plane) and TCP.C6R7.B2 (horizontal plane) was performed with squeezed noncolliding and colliding beams.

The jaws were left for a few minutes in the beam after they had reached their final inward position, to allow the losses to stabilize. Subsequently, the jaws were moved out in steps of 20 to $100 \mu \mathrm{m}$, with the next step being taken when a steady-state loss rate was observed. The beams were then brought into collisions, and the procedure was repeated. The beam intensities and left jaw collimator positions as a function of time are shown in Fig. 3. The initial and final jaw positions for each scraping in terms of the nominal beam size are provided in Table IV.

\section{Measured variables}

The halo diffusion model depends on beam intensities, beam emittances, collimator positions, and local losses, which need to be recorded. The measured variables are the following.

TABLE IV. The initial (1) and final (2) collimator jaw nominal half gaps in units of $\sigma$ for the different scrapings. The beam centers determined in beam-based alignments in March 2012 and used during operation are assumed for the calculation.

\begin{tabular}{lcc}
\hline \hline Collimator & Separated beams & Colliding beams \\
\hline TCP.D6L7.B1 (1) & $7.00 \sigma$ & $7.00 \sigma$ \\
TCP.D6L7.B1 (2) & $1.96 \sigma$ & $2.41 \sigma$ \\
TCP.C6R7.B2 (1) & $7.00 \sigma$ & $7.00 \sigma$ \\
TCP.C6R7.B2 (2) & $1.76 \sigma$ & $2.31 \sigma$ \\
\hline \hline
\end{tabular}

Intensity.-The fast beam current transformer data (FBCT) is logged at a rate of 1 and $50 \mathrm{~Hz}$.

Wire-scan emittances.-Three sets of wire scans were taken: (1) noncolliding beams, before scraping; (2) noncolliding beams, after scraping; and (3) colliding beams before scraping. Some measurements were not accurate due to saturation.

Synchrotron-light emittances. - Synchrotron-light emittance measurements were logged continuously every 3 seconds.

Collimator positions. - The left and right collimator jaw positions were logged at a rate of $1 \mathrm{~Hz}$. The jaws were kept to the zero angle throughout the study. The plots in Fig. 3 show the beam intensity and positions of the IR7 primary collimators used for scraping.

Beam losses.-The BLM data was acquired at a rate of $1 \mathrm{~Hz}(1.3 \mathrm{~s}$ integration time) and $12.5 \mathrm{~Hz}$ ( $82 \mathrm{~ms}$ integration time). The typical background signal is $2 \times 10^{-7} \mathrm{~Gy} / \mathrm{s}$, with a noise of $10^{-8} \mathrm{~Gy} / \mathrm{s}$. The signal varies from $10^{-7}$ to $10^{-3} \mathrm{~Gy} / \mathrm{s}$ (close to the beam dump threshold), as the collimator jaw cuts deeper into the beam halo.

\section{DATA ANALYSIS}

The local losses measured by the immediately downstream BLMs and the collimator jaw positions throughout the beam study are shown in Fig. 4 for the two scraping tests done for each beam. A comparison of the responses of the four BLMs immediately downstream of the collimator of interest is shown in Fig. 5. As expected, similar loss profiles with different offsets and spike amplitudes are observed for the different monitors.

\section{A. Intensity-beam loss calibration}

The BLMs measure beam losses in terms of ionizing radiation, and the resulting signal can be calibrated to determine the losses in terms of protons. Calibration factors which allow conversion from beam loss in Gy/s to $\mathrm{p} / \mathrm{s}$ are useful in determining the beam lifetime, as the BLMs allow for more precise measurements when the loss levels are low. The background losses (the $B$ parameter in the fit model) are determined during "quiet time," with no beam in the machine (between 2012-06-22, 05:28:00, and 201206-22, 05:48:00). For the local B1 BLMs the background level is $1.81 \times 10^{-6} \mathrm{~Gy} / \mathrm{s}$, and $1.06 \times 10^{-6} \mathrm{~Gy} / \mathrm{s}$ for B2.

For calibrating the response of this group of BLMs (conversion from $\mathrm{Gy} / \mathrm{s}$ to protons/s), the experiment time is subdivided into 20 -second intervals ( $40 \mu \mathrm{m}$ groupings of the collimator half gap) to obtain the average collimator position, local loss rate, and intensity decay rate. The 20 -second period was chosen to obtain a better resolution in the intensity decrease, as for some individual loss spikes (particularly at a larger jaw distance from the beam center) the intensity decrease is close to the FBCT resolution. 


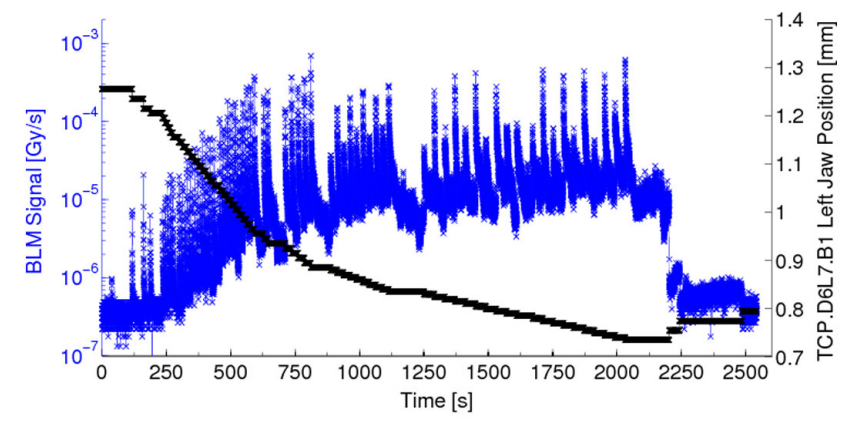

(a)BLM and TCP.D6L7.B1 left jaw positions (separated beams)

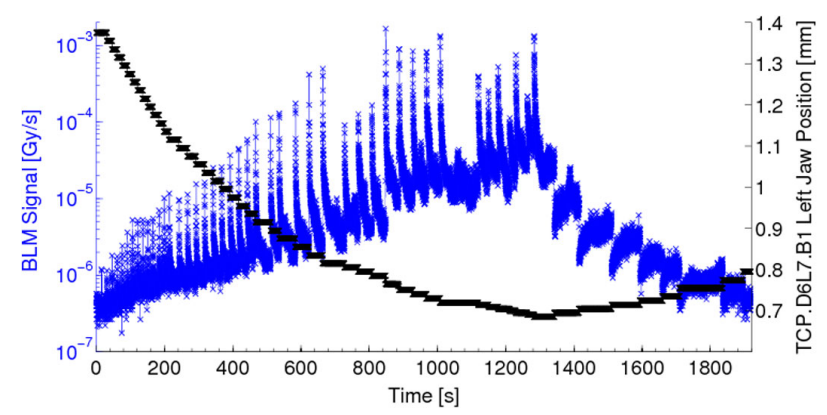

(c)BLM and TCP.D6L7.B1 left jaw positions (colliding beams)

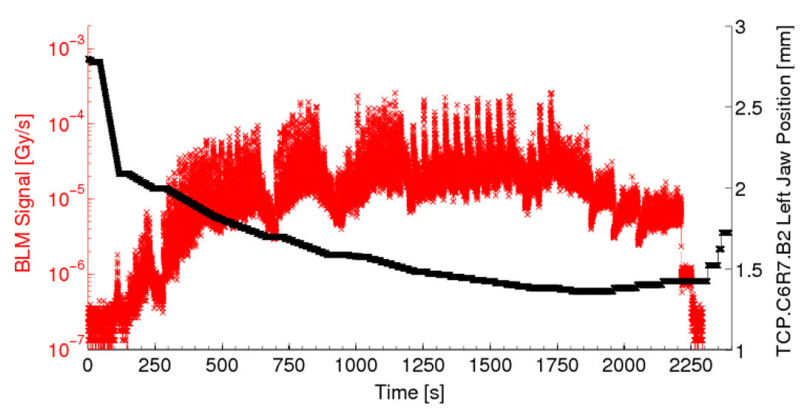

(b)BLM and TCP.C6R7.B2 left jaw positions (separated beams)

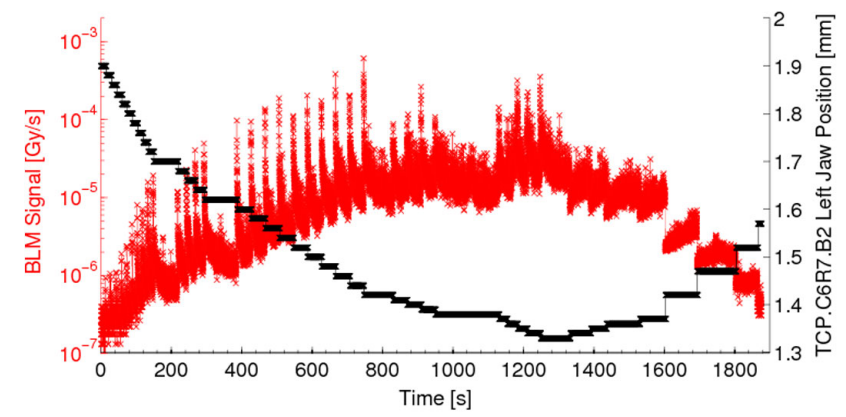

(d)BLM and TCP.C6R7.B2 left jaw positions (colliding beams)

FIG. 4. The collimator positions and associated BLM signals as a function of time with separated beams $(\mathrm{t}[0]=22.06 .2012$, 06:00:00) and colliding beams $(\mathrm{t}[0]=22.06 .2012,07: 00: 00)$.

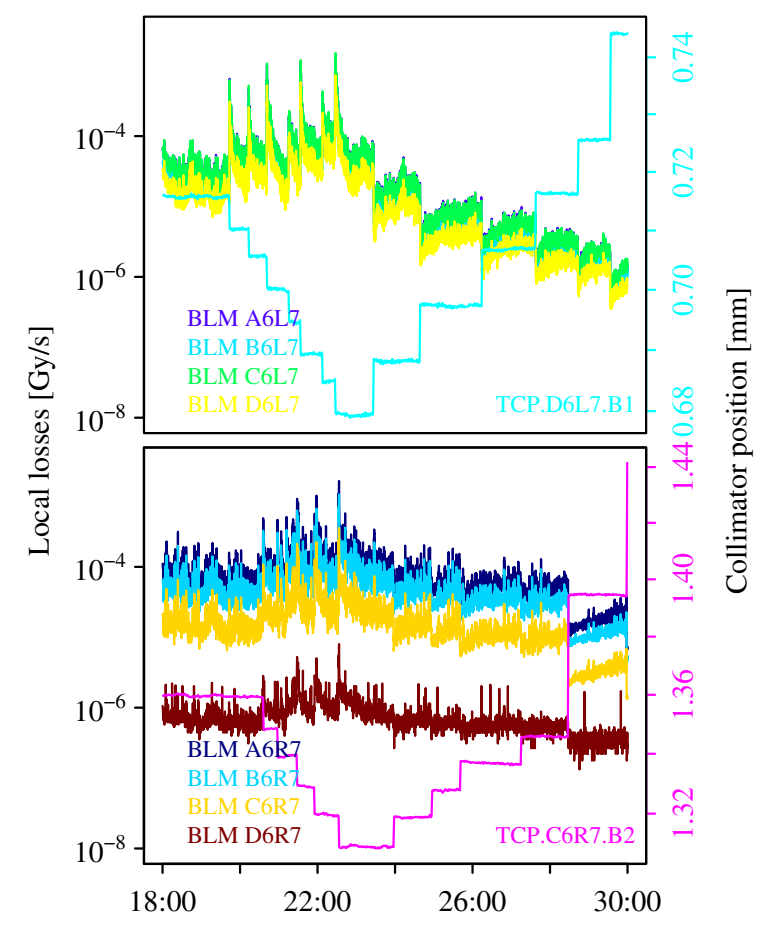

Local time [mm:ss] (t0=2012-06-22 07:18:00)

FIG. 5. Collimator jaw positions (right axis) and beam losses at different BLMs immediately downstream of the collimator used for scraping (left axis) as a function of time, for a portion of the collimator scan.
Figure 6 shows the BLM calibration constant as a function of the collimator half gap. The calibration factor, or "local loss detection efficiency," varies by more than 2 orders of magnitude. It clearly exposes the threshold when most of the losses occur locally - a half gap of $0.8 \mathrm{~mm}$ in

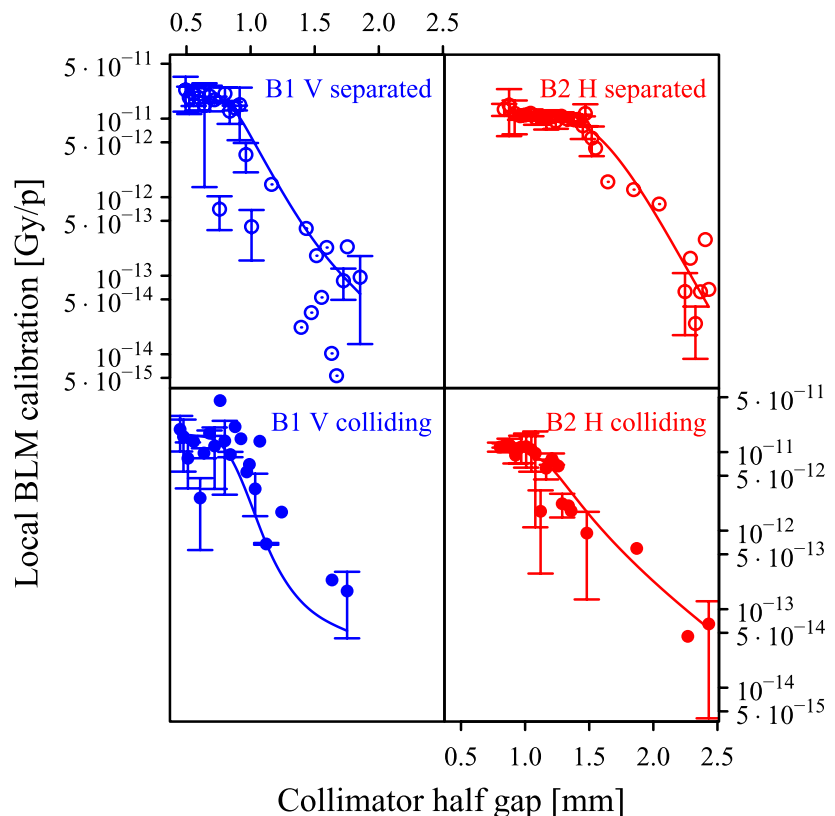

FIG. 6. Local loss monitor calibration as a function of collimator position. 


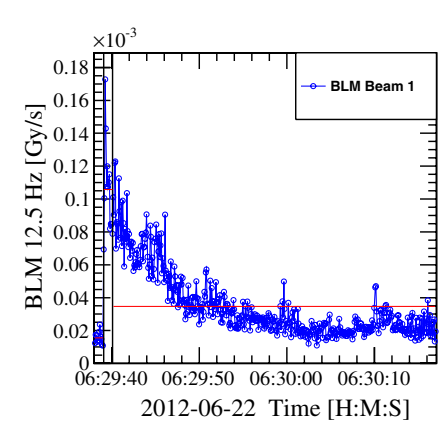

(a)BLM spike B1

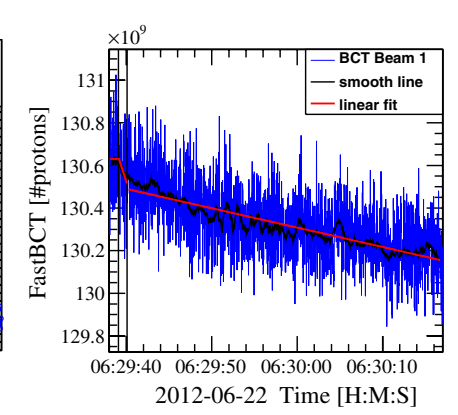

(b)FBCT signal B1

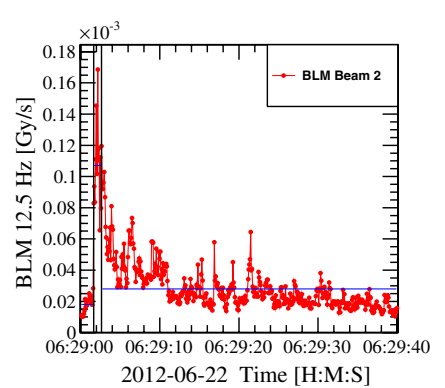

(c)BLM spike B2

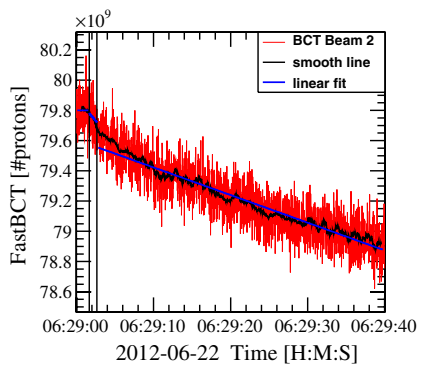

(d)FBCT signal B2

FIG. 7. Examples of fits made to the BLM and FBCT signals during loss spikes following a B1 [parts (a) and (b)] and B2 [parts (c) and (d)] collimator jaw movement, respectively. The dose in Gy is calculated by integrating the BLM signal over $\sim 1 \mathrm{~s}$ and the intensity lost in the same period is determined to calculate the calibration factor for each loss spike.

the vertical plane and $1.5 \mathrm{~mm}$ horizontally. As expected, the efficiency in collisions is lower, when a larger beam fraction is lost at the experimental insertion points. This is particularly obvious in the horizontal case. For the purposes of the diffusion analysis, these numbers are an experimental measurement of the $k$ parameter in Eq. (3). In Fig. 6, the measurements of $k$ as a function of collimator position are interpolated with a smooth spline with 4 degrees of freedom.

The dose-intensity calibration factors were also obtained using the information on the BLM spikes over intervals of $1 \mathrm{~s}$ from the jaw movement, with the BLM taken to be the one downstream closest to the collimator. The advantage in this case is that any intensity decrease caused by beam losses at the collimator can be directly correlated to the loss spike under study. This type of analysis is more sensitive to "beam cleaning," i.e., the fast, multiturn beam losses caused by an inward collimator step that would be present even in a linear machine and in the absence of diffusion processes. The BLM and intensity data were extracted for each loss spike interval. The dose in Gy was calculated as the average loss signal for the peak integrated over $\sim 1 \mathrm{~s}$, as shown in Figs. 7(a) and 7(c). The intensity lost over the same time period was also determined [see examples in
Figs. 7(b) and 7(d)]. The particle loss was calculated as a percentage of the intensity recorded by the FBCT before the loss spike, and is shown as a function of the jaw gap in mm in Figs. 8(a) and 8(b).

The calibration factors in Gy/p are shown in Fig. 9. The values compare well with those found during the 2011 scraping study for the same collimators $(8.3 \times$ $10^{-13} \mathrm{~Gy} / \mathrm{p}$, see [11]). The apparent difference in scale between Figs. 9 and 6 is because the former plot takes into account only the closest BLM to the collimator, while the latter considers the four closest downstream BLMs. The particle loss was calculated for a given jaw half gap by integrating over the static Gaussian beam distribution for the jaw step size used. The nominal beam sizes at the collimator locations are considered for the beam distribution calculation.

Figures 8(c) and 8(d) show the BLM signal in arbitrary units, which is calculated by multiplying the ratio of the dose to the intensity before the jaw movement by the average calibration factor of $1.2 \times 10^{12} \mathrm{p} / \mathrm{Gy}$ and an additional factor. The additional factor $(\sim 0.65)$ was necessary to scale the resulting BLM signal to the static distribution curves, which are the same as in Figs. 8(a) and 8(b), and derives from an offset between the FBCT signal and the

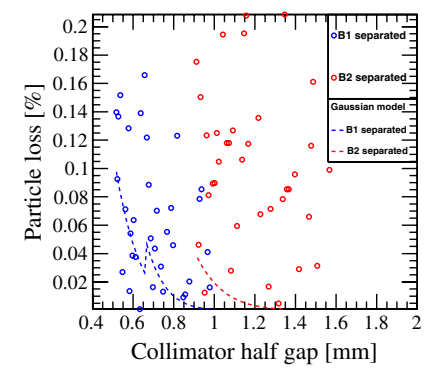

(a)Particle loss (separated beams)

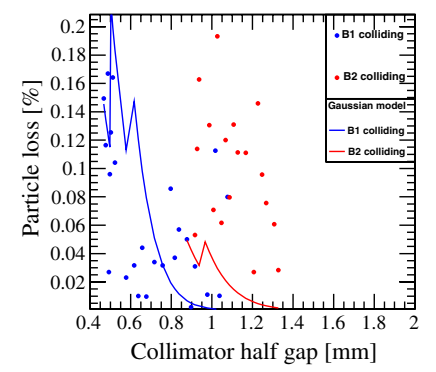

(b)Particle loss (colliding beams)

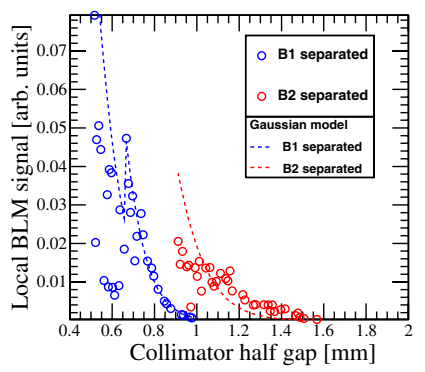

(c)Local BLM signal (separated beams)

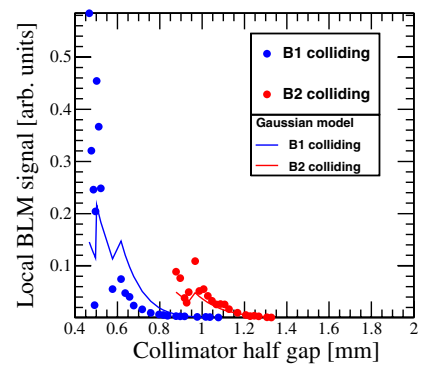

(d)Local BLM signal (colliding beams)

FIG. 8. Particle loss as a percentage of the intensity measured before the loss spike [parts (a) and (b)] and local BLM signal [parts (c) and (d)], with comparison to the static beam distribution at the start of scraping. The discontinuities in the static distribution curves are due to different jaw step sizes being used throughout the beam study. 


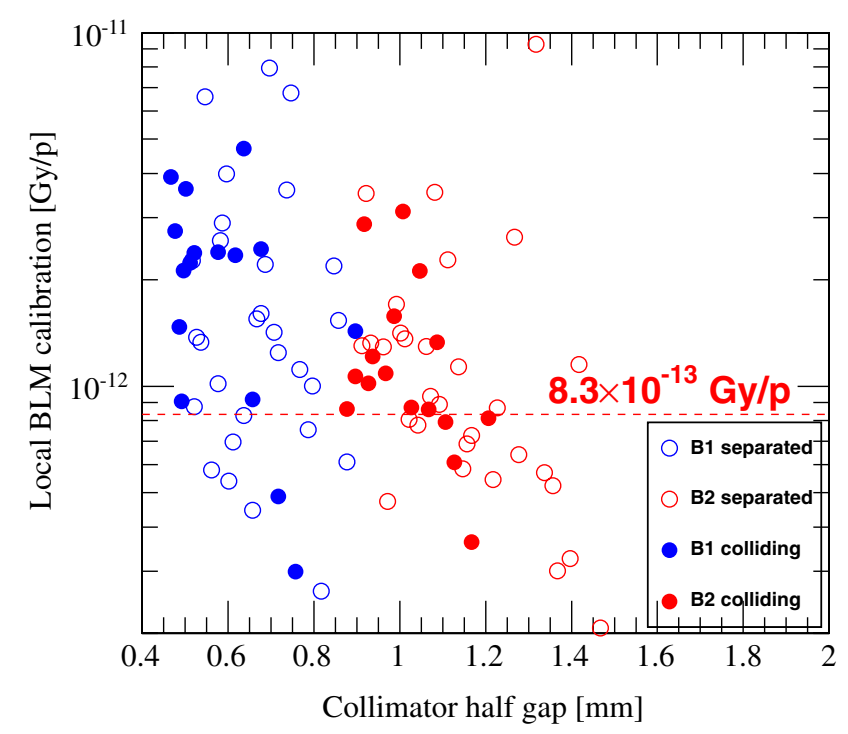

FIG. 9. The calibration factors calculated for each loss spike in units of $\mathrm{Gy} / \mathrm{p}$, as a function of the collimator half gap in $\mathrm{mm}$. The red dotted line indicates the calibration factor found in the 2011 scraping study for reference.

actual intensity in the LHC. The discontinuities in the static distribution curves are due to different jaw step sizes being used throughout the beam study.

\section{B. Evolution of beam emittance}

The objective of measuring the beam emittances is to determine the beam sizes at the collimator positions and estimate the core diffusion rates. The initial emittances are calculated from the wire scan (see Table V) and the synchrotron-light data. Both wire-scan and synchrotronlight emittance measurements are affected by the scraping of the tails (Fig. 10). The emittances measured at the start of the beam study (2012-06-22, 06:08:00) are used to convert the collimator gaps into beam sigmas. The statistical uncertainty is calculated from the difference between inward and outward wire movement. The average of the synchrotron-light measurements is used, with a statistical uncertainty deduced from the spread in data points.

The final emittance value is obtained by averaging wire scan and synchrotron-light data. Its error is the combination in quadrature of the statistical errors plus a systematic error due to the difference between the two techniques. Results are reported in Table VI. The beam sizes at the

TABLE V. Normalized, $1 \sigma$ wire-scan emittances: (1) noncolliding beams before scraping, (2) noncolliding beams after scraping, and (3) colliding beams before scraping.

\begin{tabular}{lccccc}
\hline \hline Type & Time & $\epsilon_{x}^{B 1}[\mu \mathrm{m}]$ & $\epsilon_{y}^{B 1}[\mu \mathrm{m}]$ & $\epsilon_{x}^{B 2}[\mu \mathrm{m}]$ & $\epsilon_{y}^{B 2}[\mu \mathrm{m}]$ \\
\hline$(1)$ & $05: 53$ & 1.92 & 1.36 & 1.69 & 1.73 \\
$(2)$ & $06: 47$ & 1.80 & 1.23 & 1.20 & 1.21 \\
$(3)$ & $07: 01$ & 1.71 & 1.45 & 1.28 & 1.26 \\
\hline \hline
\end{tabular}

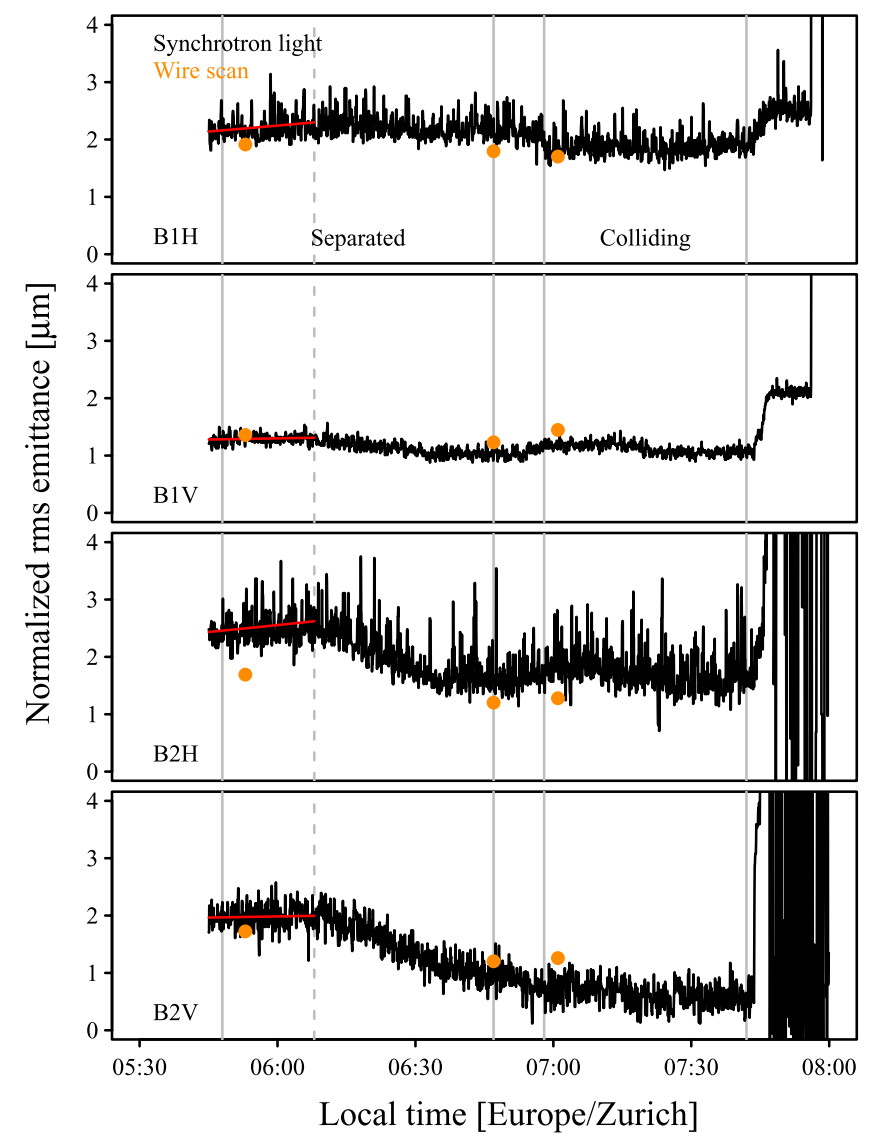

FIG. 10. Synchrotron-light and wire-scan emittances over the course of the study.

collimators calculated from the initial emittances are $0.156 \mathrm{~mm}$ (B1 vertical) and $0.270 \mathrm{~mm}$ (B2 horizontal). The emittance growth rates $\gamma=\dot{\epsilon} / \epsilon$ are deduced from the slope of the synchrotron-light data before 2012-06-22, 06:08:00. According to the diffusion model, the slope of the diffusion coefficient in the beam core is related to the growth rate of the geometrical emittance: $D^{\prime}=$ $d D / d J=\dot{\epsilon}$. These slopes are presented in Table VI. In Sec. V, diffusion coefficients calculated from these slopes $\left(D=D^{\prime} \cdot J=\dot{\epsilon} \cdot J\right)$ are compared with the measured diffusion coefficients in the halo. One would expect halo diffusion to be at least as large as core diffusion, or larger if there are additional diffusion mechanisms at increasing amplitudes, such as magnet nonlinearities or resonances.

\section{Parametric fits of the diffusion model}

The model described in Sec. II is used to perform fits to the inward and outward jaw movements separately. The initial values for the fit parameters are determined as follows. The steady-state rates are evaluated by averaging the losses before the step $(-0.5 \mathrm{~s}$ from the step or earlier) and after (4 $\mathrm{s}$ after or later). The starting point for the diffusion coefficient is the value obtained from the decay $3 \mathrm{~s}$ after the step (where the losses are $\approx 1 / \sqrt{t}$ ). The time 
TABLE VI. Initial emittances (wire scan, synch-light, average), emittance growth rates (EGR) $\gamma$, and diffusion slopes $D^{\prime}$.

\begin{tabular}{|c|c|c|c|c|c|c|c|c|c|c|}
\hline \multirow[b]{2}{*}{ Plane } & \multirow[b]{2}{*}{$\epsilon_{w s}$} & \multicolumn{3}{|c|}{ Emittance $[\mu \mathrm{m}]$} & \multirow[b]{2}{*}{$\epsilon$} & \multirow[b]{2}{*}{$\delta \epsilon$} & \multicolumn{2}{|c|}{ EGR $[1 / \mathrm{s}]$} & \multicolumn{2}{|c|}{ Diffusion $[\mu \mathrm{m} / \mathrm{s}]$} \\
\hline & & $\delta \epsilon_{w s}$ & $\epsilon_{s l}$ & $\delta \epsilon_{w s}$ & & & $\gamma$ & $\delta \gamma$ & $D^{\prime}$ & $\delta D^{\prime}$ \\
\hline B1 H & 1.92 & $1.3 \times 10^{-2}$ & 2.15 & $9.6 \times 10^{-3}$ & 2.03 & 0.07 & $5.23 \times 10^{-5}$ & $1.50 \times 10^{-5}$ & $2.49 \times 10^{-8}$ & $7.4 \times 10^{-9}$ \\
\hline $\mathrm{B} 1 \mathrm{~V}$ & 1.36 & $2.0 \times 10^{-3}$ & 1.29 & $4.7 \times 10^{-3}$ & 1.33 & 0.02 & $1.70 \times 10^{-5}$ & $1.00 \times 10^{-5}$ & $5.27 \times 10^{-9}$ & $3.2 \times 10^{-9}$ \\
\hline $\mathrm{B} 2 \mathrm{H}$ & 1.69 & $6.6 \times 10^{-3}$ & 2.43 & $1.3 \times 10^{-2}$ & 2.06 & 0.21 & $5.37 \times 10^{-5}$ & $1.90 \times 10^{-5}$ & $2.60 \times 10^{-8}$ & $9.6 \times 10^{-9}$ \\
\hline B2 V & 1.73 & $4.6 \times 10^{-2}$ & 1.98 & $1.2 \times 10^{-2}$ & 1.85 & 0.09 & $1.10 \times 10^{-5}$ & $1.70 \times 10^{-5}$ & $4.79 \times 10^{-9}$ & $7.2 \times 10^{-9}$ \\
\hline
\end{tabular}

of the step, $t_{0}$, is known from the time stamps of the collimator position (with $1 \mathrm{~s}$ resolution) and from the increase in the losses ( $80 \mathrm{~ms}$ resolution). The duration of the step $\Delta t$ is estimated from the step size and the nominal jaw speed, $2 \mathrm{~mm} / \mathrm{s}$. Step time and duration can also be left as free parameters within some reasonable limits.

The distribution of the response variable (loss rate) is not Gaussian, as one can see for instance from Fig. 5, because of instrumental effects (such as beam jitter and mechanical vibrations) and because of the Poissonian nature of the shower process. For this reason, fit parameters are determined by minimizing not a $\chi^{2}$, but the sum of absolute deviations of the model from the data (robust estimation). For the same reason, a first estimate of the statistical errors comes from the Hessian matrix of the $\chi^{2}$ function, but the final error analysis is done by bootstrapping. For each step, bootstrapping is done by resampling the data points with replacement and repeating the fit several times with the same initial parameters. The final results are the median of the fit results and the uncertainties come from their spread.

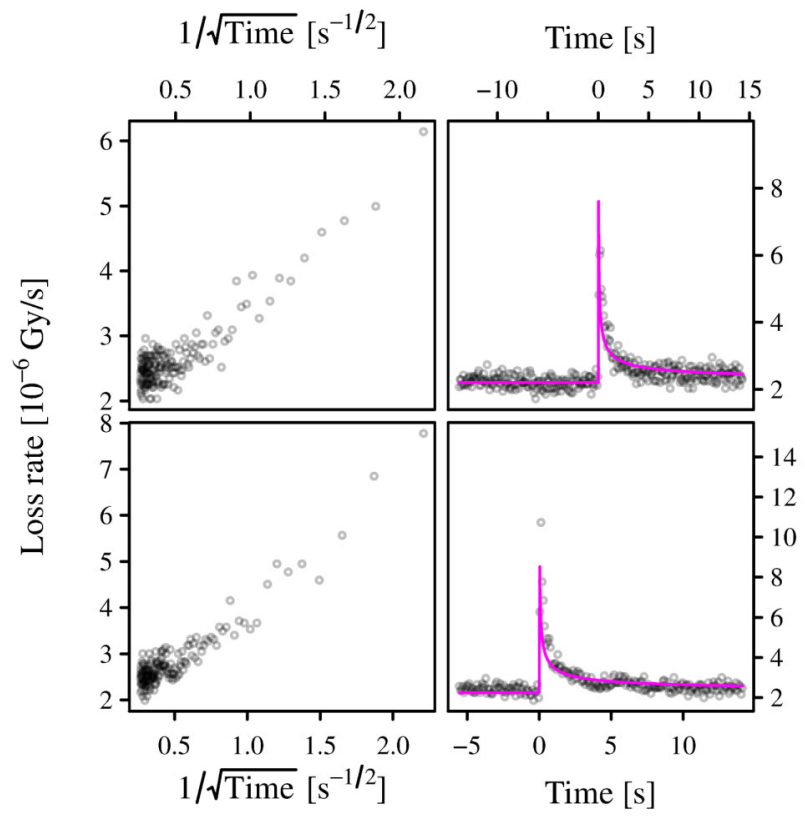

(a)Inward collimator jaw movement
Examples of the fit results are plotted in Fig. 11 for inward and outward jaw movements. Not all fits converged: in some cases, the model cannot explain the first few seconds of losses after the step. This seems to be due to the losses decaying differently from $1 / \sqrt{t}$, as shown in the first column of the plots. This fact could be a combination of instrumental effects (the details of the collimator movement) and pure beam removal/cleaning. But in general, the model seems to reproduce the main features of the loss evolution with time quite well. Less data is available for the outward movement, as the losses were found to quickly decrease to the background level when a half gap of $2.2 \sigma$ for $\mathrm{B} 1$ and $2.8 \sigma$ for $\mathrm{B} 2$ was reached.

\section{RESULTS}

The diffusion coefficients as a function of action are shown in Fig. 12 for all four cases: horizontal and vertical, each with separated or colliding beams. With separated beams, the diffusion in the $\mathrm{B} 2$ horizontal plane is higher than that in the B1 vertical plane. In the horizontal plane,

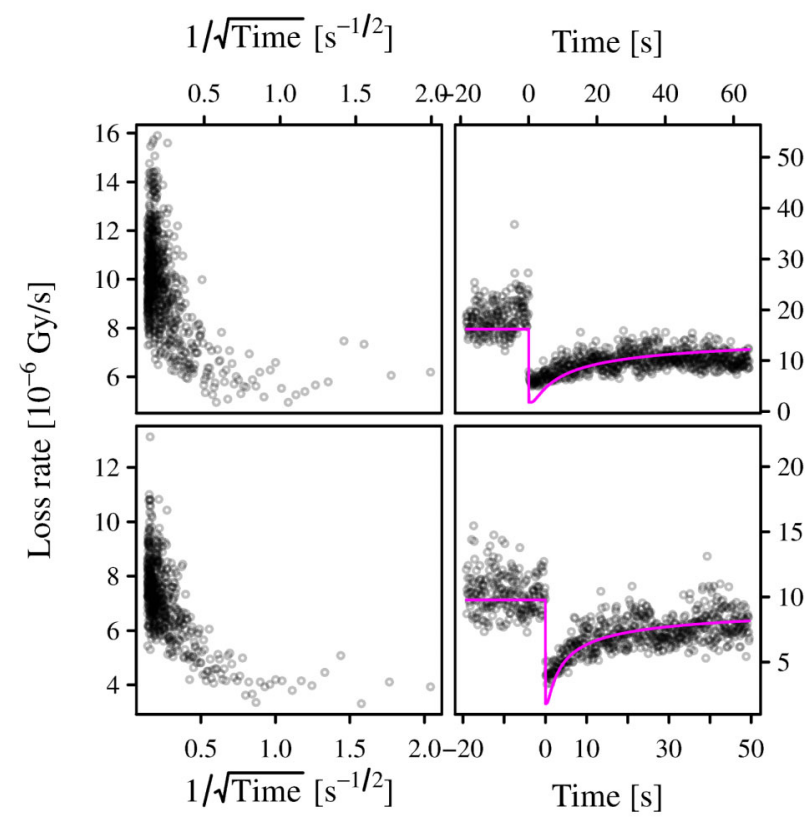

(b)Outward collimator jaw movement

FIG. 11. Examples of loss-rate responses to inward (left panel) or outward (right panel) collimator steps. In each panel, the plots on the left side show the decay as a function of $1 / \sqrt{t}$. The data and the diffusion model fit as a function of time are shown in the plots on the right side of each panel. 


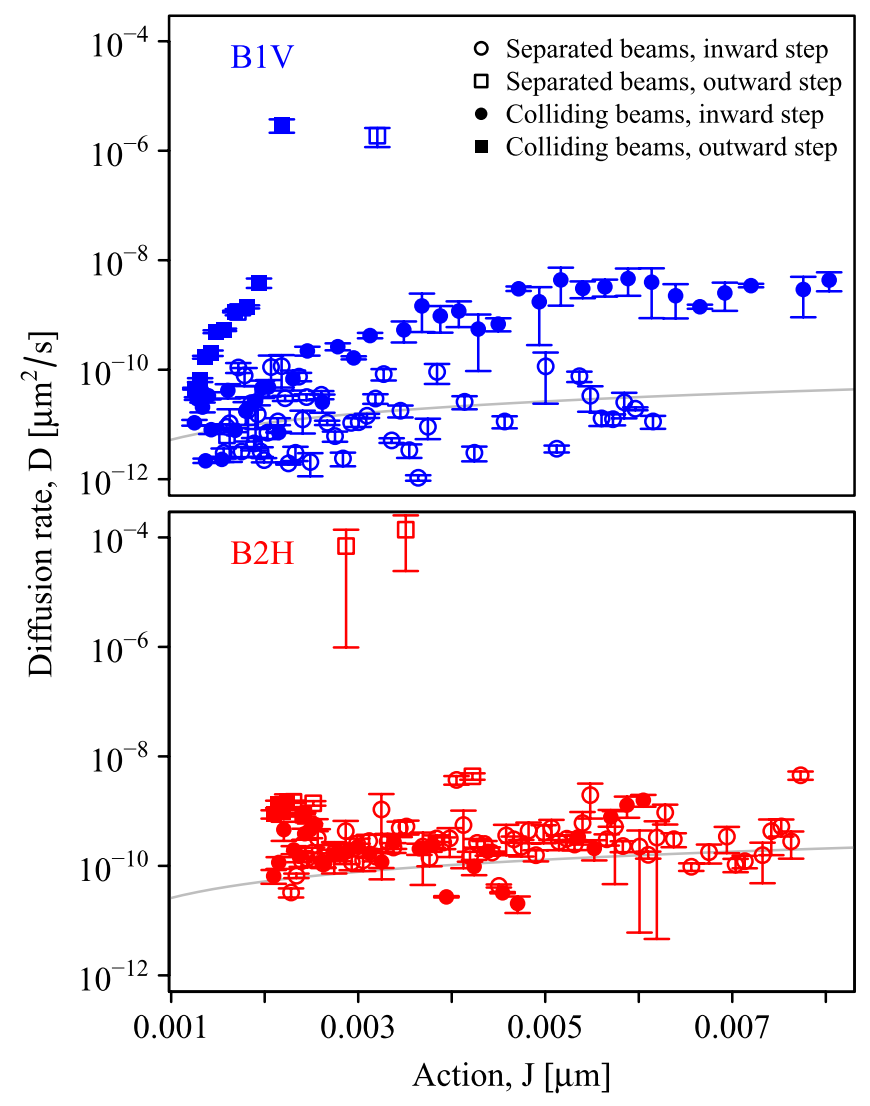

FIG. 12. Diffusion coefficient as a function of action from the collimator scan (points), compared with the expectation from core emittance growth rates (lines).

there seems to be little difference between the separated and colliding cases, while in the vertical plane, collisions enhance diffusion by about 2 orders of magnitude. The reason for this is not fully understood. A possible explanation is due to the larger emittances measured in the horizontal plane in B1. If there is sufficient coupling, with the beams in collisions, diffusion could be enhanced also in the vertical plane in B1, as B2 has lower emittances. Unfortunately, collimator scans could not be performed for using the horizontal B1 and vertical B2 collimators, and hence no generalizations can be drawn from a lack of measurements.

A significant systematic effect is the larger diffusion rates obtained from the outward steps. They are not understood, but are probably due to the fact that, after scraping, a different beam population is being sampled. A comparison of inward and outward steps was motivated both by practical and physical reasons. Practical considerations included checking for instrumental systematic effects. Moreover, outward steps should be independent of fast, multiturn particle removal or perturbations of the beam density distribution induced by the collimators. From the physical point of view, if phase space contains particles trapped in islands and motion is not completely stochastic, one may expect different removal and repopulation rates for a given amplitude. In practice, these effects are difficult to disentangle.

The grey curves in Fig. 12 are not an interpolation of the data: they represent the dependence $D(J)$ that one would obtain from the core emittance growth rates (Table VI). This comparison allows one to draw several conclusions: (1) the diffusion rates measured with the collimator scan technique have reasonable values; (2) without collisions, the beam halo in the LHC diffuses almost like the core up to large amplitudes; (3) no significant dynamic aperture effects (sharp rises in diffusion rate) are observed in the amplitude range explored in these experiments.

From the diffusion coefficients as a function of amplitude, one can calculate the distribution of impact parameters, i.e., the depth at which particles impinge on the collimators. These distributions can affect the efficiency of a collimator system [6]. Impact parameters from diffusion coefficients can be calculated in two ways [14]. The first uses a Monte Carlo technique to generate particles near a collimator, propagating their trajectories including diffusion, and calculating their distributions when they reach the collimators. The second method uses an analytical form of the impact parameter distribution based on the diffusion phenomenology. In both cases, the average impact parameter is found to scale with the diffusion coefficient as $D^{1 / 2}$. Although the detailed analysis is beyond the scope of this paper, we obtain average impact parameters in the range between 0.02 to $0.3 \mu \mathrm{m}$ for diffusion coefficients of $1.2 \times 10^{-11}$ to $3.6 \times 10^{-9} \mu \mathrm{m}^{2} / \mathrm{s}$, for actions of 0.002 to $0.008 \mu \mathrm{m}$. These results are consistent with the assumptions commonly used for the LHC collimation system design and performance evaluation [15].

\section{CONCLUSIONS}

For the first time in the LHC, transverse beam halo diffusion rates were measured. This was done by observing the response of beam loss rates to small inward or outward collimator steps. Halo dynamics appeared to be stochastic in most cases. Occasional systematic deviations in the first few seconds after the collimator step were observed, probably due to the details of collimator movements and to multiturn halo removal. Separated beams exhibited a slower halo diffusion than colliding beams, comparable with the emittance growth from the core. No dynamic aperture effects, such as dramatic increases in diffusion coefficient, were observed up to about $7 \sigma$ in transverse amplitude. This can be interpreted as a confirmation of the extremely good quality of the magnetic fields in the machine. Collisions enhanced halo diffusion in the vertical plane by about 2 orders of magnitude. From the measured diffusion coefficients, it is possible to estimate the equilibrium tail populations and the distribution of impact parameters on the primary collimator jaws. These measurements give experimental information on the relationship between 
halo population and halo dynamics, emittance growth, beam lifetime, and collimation efficiency. They are also important inputs for collimator system design and upgrades.

\section{ACKNOWLEDGMENTS}

The authors would like to thank S. Cettour Cave, A. Macpherson, D. Jacquet, and M. Solfaroli Camillocci, the LHC operators on shift during the beam study. Gratitude is also expressed towards R. De Maria for providing a script to acquire the intensity data at $50 \mathrm{~Hz}$, and to X. Buffat, W. Herr, B. Holzer, T. Pieloni, and J. Wenninger for discussions on the contribution of beam-beam effects on beam diffusion. Fermilab is operated by Fermi Research Alliance, LLC under Contract No. DE-AC02-07CH11359 with the United States Department of Energy. This work was partially supported by the U.S. LHC Accelerator Research Program (LARP).

[1] A. J. Lichtenberg and M. A. Lieberman, Regular and Chaotic Dynamics (Springer-Verlag, New York, 1992), p. 320 .

[2] T. Chen et al., Phys. Rev. Lett. 68, 33 (1992).

[3] A. Gerasimov, Report No. FERMILAB-PUB-92-185, 1992.

[4] F. Zimmermann, Part. Accel. 49, 67 (1995) [https:// cds.cern.ch/record/274807/files/p67.pdf ].
[5] T. Sen and J.A. Ellison, Phys. Rev. Lett. 77, 1051 (1996).

[6] R. W. Aßmann et al., in Proceedings of the 8th European Particle Accelerator Conference, Paris, France, 2002 (EPS-IGA and CERN, Geneva, 2002), pp. 197-199.

[7] B. Dehning et al., in Proceedings of PAC'07, Albuquerque, NM, 2007 (IEEE, New York, 2007), pp. 4192-4194.

[8] K. H. Mess and M. Seidel, Nucl. Instrum. Methods Phys. Res., Sect. A 351, 279 (1994).

[9] R. W. Aßmann, E. Holzer, J.-B. Jeanneret, V. Kain, S. Redaelli, G. Robert-Demolaize, and J. Wenninger, in Proceedings of the 9th European Particle Accelerator Conference, Lucerne, Switzerland, 2004 (EPS-AG, Lucerne, 2004), pp. 1825-1827.

[10] G. Valentino, R.W. Aßmann, R. Bruce, S. Redaelli, A. Rossi, N. Sammut, and D. Wollmann, Phys. Rev. ST Accel. Beams 15, 051002 (2012).

[11] F. Burkart, R.W. Aßmann, R. Bruce, M. Cauchi, D. Deboy, L. Lari, S. Redaelli, A. Rossi, D. Wollmann, and G. Valentino, in Proceedings of IPAC'11, San Sebastian, Spain, 2011 (EPS-AG, Spain, 2011), pp. 3756-3758.

[12] G. Stancari, G. Annala, T. R. Johnson, D. A. Still, and A. Valishev, in Proceedings of IPAC'11, San Sebastian, Spain, 2011 (Ref. [11]), pp. 1882-1884.

[13] G. Stancari, arXiv:1108.5010.

[14] M. Seidel, DESY Report No. 94-103, 1994.

[15] R.W. Aßmann, J.-B. Jeanneret, and D. Kaltchev, in Proceedings of APAC'01, Beijing, China, 2001 (Institute of High Energy Physics, Beijing, 2001), pp. 204-208. 